\title{
Iterative Method for Constructing Dosing Regimes for Controlling HIV Dynamics
}

\author{
N. D. Evans \\ School of Engineering, University of Warwick, Coventry, CV4 7AL, United Kingdom \\ E-mail: Neil.Evans@warwick.ac.uk \\ Sci Pharm. 2010; 78: 588 \\ doi:10.3797/scipharm.cespt.8.LPM01
}

A pharmacokinetic (PK) model describes the absorption, distribution, metabolism and excretion of a pharmaceutical, thus providing, once properly validated, quantitative predictions of the consequences of various dosing regimens. Pharmacological activity might be linked to properties of a time course for a particular compartment (such as half-life or area-under-curve, AUC), or directly modelled by coupling the PK model with a pharmacodynamic (PD) model of the physiological effect of the agent.

Due to the high replication and mutation rates of the human immunodeficiency virus (HIV), which ultimately results in strains resistant to any single agent, a multiple-agent strategy has to be adopted. Highly active anti-retroviral therapy (HAART) uses two or more agents that target different components of the HIV replication cycle, but these agents typically produce adverse side effects in patients. A number of authors have applied techniques from nonlinear control to this problem, but in general the explicit link between the drug dose and the physiological effect, via the PK, is neglected so that the controls derived are continuous in time. In [1] the control problem is addressed via feedback linearization applied to a PD model of HIV dynamics. A single-compartment PK model is then used to estimate a dosing regime that gives rise to the required continuous control law.

In [2] a fixed-point approach was taken to determine the doses required to obtain a particular AUC or target profile. Although a constructive method is shown for determining the required dosing scheme, full solution trajectories and their integrals are required. An iterative method for constructing the dosing regime is proposed here that provides the fixed-point solution from [2] but is computationally easier to implement, being based on the underlying system equations. After each iteration the current dosing scheme is updated based on a linear operator applied to the difference between achieved and desired target vectors.

The iterative method is applied to the coupled PK-PD model from [1] in order to directly construct a dosing regime, rather than indirectly via a continuous efficacy profile.

[1] Mhawej MJ, Moog CH, Biafore F, Brunet-François C. Control of the HIV infection and drug dosage. Biomed Signal Process Control. 2010; 5: 45-52. doi:10.1016/j.bspc.2009.05.001

[2] Evans ND. Optimal oral drug dosing via application of the Contraction Mapping Theorem. Biomed Signal Process Control; in press. 